\title{
The characterization of Bordetella pertussis strains isolated in the Central-Western region of Brazil suggests the selection of a specific genetic profile during 2012-2014 outbreaks
}

\author{
E. L. ROCHA ${ }^{1}$, D. LEITE ${ }^{2}$, C. H. CAMARGO ${ }^{2}$, L. M. MARTINS ${ }^{2}$, \\ R. S. N. SILVA ${ }^{3}$, V. P. MARTINS ${ }^{1}$ AND T. A. CAMPOS ${ }^{1 *}$ \\ ${ }^{1}$ Departamento de Biologia Celular, Instituto de Ciências Biológicas, Universidade de Brasília, Campus \\ Universitário 'Darcy Ribeiro', Brasília, DF, Brasil \\ ${ }^{2}$ Laboratório de Referência Nacional para Pertussis, Instituto Adolfo Lutz, São Paulo, SP, Brasil \\ ${ }^{3}$ Laboratório de Bacteriologia do Laboratório Central de Saúde Pública do Distrito Federal, Brasília, DF, Brasil
}

Received 25 May 2016; Final revision 6 December 2016; Accepted 16 December 2016; first published online 21 February 2017

\section{SUMMARY}

Pertussis is a worldwide acute respiratory disease caused by the bacterium Bordetella pertussis. Despite high vaccine coverage, the bacterium continues to circulate in populations and is still one of the most common vaccine-preventable diseases. In Brazil, pertussis incidence has presented a significant decrease since 1990 but since 2011 a sudden increase in incidence has been observed. Thus, the aim of this study was to perform a molecular epidemiological characterization of $B$. pertussis strains isolated in the Central-Western region (specifically in Distrito Federal) of Brazil from August 2012 to August 2014. During this period, 92 B. pertussis strains were isolated from the outbreaks. All strains were characterized by serotyping and $X b a \mathrm{I}$ pulsed-field gel electrophoresis profiles. From August to December 2012, the most prevalent serotype observed was 1,3 (13/17). During 2013 the prevalence of serotype 1,3 decreased (13/30) and from January 2014 to August 2014 the most prevalent serotype was 1,2 (33/45). Fourteen PFGE profiles were identified. Of these, BP-XbaI0039 prevalence increased from 3/17 in 2012 to $10 / 30$ in 2013, and $35 / 45$ in 2014. These results evidence the selection of a specific genetic profile during this period, suggesting the occurrence of a bacterial genomic profile with high circulation potential.

Key words: Bordetella pertussis, genetic profile selection, pertussis, whooping cough.

\section{INTRODUCTION}

Pertussis, or whooping cough, caused by the bacterium Bordetella pertussis is a worldwide acute respiratory disease [1]. Despite the implementation of immunization programmes and high vaccine coverage, the bacterium continues to circulate in populations and is still one of the most common vaccine-preventable

\footnotetext{
* Author for correspondence: Dr T. A. Campos, Departamento de Biologia Celular, Instituto de Ciências Biológicas, Universidade de Brasília. Campus Universitário 'Darcy Ribeiro', Asa Norte, CEP 70910-900, Brasília, DF, Brasil.

(Email address: tatianadecampos@unb.br)
}

diseases [2]. The disease is severe in newborns and unvaccinated infants, and can cause mortality in these age groups. Moreover, the absence or low coverage of adult boosters in some countries increases the circulation of the bacterium and enhances the transmission to unimmunized infants. Recently, large outbreaks of pertussis have been reported in various countries from America and Europe. These outbreaks indicate that $B$. pertussis strains are still circulating among people and causing epidemics even in highly vaccinated populations [3].

In Brazil, pertussis vaccination was introduced in 1977. In 2004, a booster dose vaccination was introduced for children aged 4-6 years. Currently, three 
primary doses are administered to babies at ages 2, 4 and 6 months, with two booster doses recommended at ages 15 months and 4 years. The Brazilian pertussis vaccination programme uses whole cell $(\mathrm{wP})$ vaccine for the first dose and for the booster doses. Acellular (aP) vaccine is only recommended for pregnant women and for children with severe risk of adverse reactions to $\mathrm{wP}$ vaccination [4].

Since 1990, pertussis incidence has shown a significant decrease, from $10 / 100000$ population to $0 \cdot 32 / 100$ 000 population in 2010 , with 427 confirmed cases. Markedly, since 2011 a sudden increase in incidence has been observed: 2070 cases were reported in that year, reaching 8447 confirmed cases in the country during 2014 [4]. The main hypotheses to explain the recurrence of pertussis outbreaks are the genetic and phenotypic changes of the strains, the low potential for induction of immunity by the vaccine, the improvement of both epidemiology surveillance and diagnostic techniques $[5,6]$.

To contain pertussis resurgence, effort must be made to better understand and discontinue the mechanisms associated with the bacterial dissemination. The characterization of the circulating strains may provide insights about bacterial mechanisms associated with the success of the transmission. In this way, the aim of this study was to perform the molecular epidemiological characterization of $B$. pertussis strains isolated in the Distrito Federal region of Brazil from July 2012 to August 2014

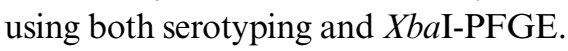

\section{METHODS}

\section{Bacterial isolation and growth conditions}

B. pertussis strains were isolated from nasopharyngeal swabs of patients presenting whooping cough signs during August 2012 to August 2014 in the Distrito Federal region of Brazil (Central-Western Region of Brazil). The isolation was done by the Central Public Health Laboratory of the Federal District (Laboratório Central de Saúde do Distrito Federal; LACEN), the official surveillance institute of the region. The samples used in this study were obtained from the epidemiology surveillance collection of LACEN. Besides the isolates, information about patients' gender, patients' age and period of the strains isolation were used (Supplementary Table S1). All strains isolated during the period analysed were used.

Briefly, nasopharyngeal swabs were cultured in Regan-Lowe agar plates (Oxoid, UK) supplemented with $10 \%$ sheep blood and $40 \mu \mathrm{g} / \mathrm{ml}$ cefalexin (Sigma Aldrich, USA). The plates were incubated under humid conditions at $35-37^{\circ} \mathrm{C}$ for up to 10 days. Suggestive $B$. pertussis colonies were submitted for identification on the VITEK-2 system (bioMeriéux, France). All positive cultures of $B$. pertussis were maintained at $-70{ }^{\circ} \mathrm{C}$. For serotyping and genetic similarity analysis by pulsed field gel electrophoresis (PFGE), all B. pertussis isolates were cultivated in Regan-Lowe plates supplemented with $10 \%$ sheep blood under humid conditions at $35-37^{\circ} \mathrm{C}$ during 3 or 4 days.

\section{Serotyping}

Serotyping was done by agglutination test for O1-specific B. pertussis and for Fim 2 and Fim 3 fimbrial antigens by the slide agglutination test using $\mathrm{O} 1$, and Fim 2 and Fim 3 antibodies, respectively [7, 8]. The bacterial suspension used was done in saline solution $0 \cdot 85 \%$. B222 and B201 B. pertussis strains were used as positive controls for serotypes 1,2 and 1,3 [7]. The $\mathrm{O} 1$ antiserum and fimbrial 2 and 3 monoclonal antibodies were prepared at Instituto Adolfo Lutz using reference strains kindly supplied by the Pertussis Reference Laboratory, University of Manchester.

\section{PFGE}

PFGE analysis was performed as described by Advani et al. [9], using $X b a \mathrm{I}$ as a restriction enzyme, with the following modifications. Electrophoresis was performed at $6 \mathrm{~V} / \mathrm{cm}$ for $24 \mathrm{~h}$ at $14{ }^{\circ} \mathrm{C}$ and pulse times of $5-6 \mathrm{~s}$ for $11 \mathrm{~h}$ and $8-35 \mathrm{~s}$ for $13 \mathrm{~h}$. The PFGE profiles were analysed using BioNumerics software package v. 4.0 (Applied Maths Inc., USA). The profiles were compared by the unweighted pair-group method using arithmetic averages (UPGMA) as the clustering method, with $1 \%$ band tolerance and $1 \%$ optimization settings with Dice similarity coefficient. Three strains were not used in PFGE because they did not grow.

\section{Statistical analysis}

Statistical analysis was performed using SPSS software v. 18.0 (IBM Corp., USA). The $\chi^{2}$ test was used for proportion comparison.

\section{Ethical approval}

The study was conducted as an IRB-approved protocol (Ethical approval no. CAAE 43098415.6.1001.5553- 
FEPECS/SES-DF Secretaria de Saúde do Distrito Federal, DF, Brazil).

\section{RESULTS AND DISCUSSION}

In Brazil, suspected pertussis cases are confirmed or discarded at the health unit on the basis of clinical, epidemiological or laboratory criteria. A case with a negative or absence of bacterial culture, lack of epidemiological link, or without confirmation of other aetiology is confirmed by clinical criteria if the blood count presents: $\geqslant 20000$ leukocytes $/ \mu 1$ and $\geqslant 10000$ lymphocytes $/ \mu$ l. Epidemiological criteria are met with a confirmed case of pertussis via laboratory testing, which is performed between the beginning of the catarrhal period until 3 weeks after the beginning of the disease paroxysmal period. Laboratory criteria are based on the isolation of $B$. pertussis using a culture of nasopharyngeal secretion or positive polymerase chain reaction [4]. In the region analysed in this study, 92 B. pertussis strains were isolated from August 2012 to August 2014. The majority of strains were obtained in 2014 (January-August; 45/92), followed by the year 2013 (30/92), and by 2012 (August-December; 17/92). The most isolates were obtained from patients aged 24 months (40/92), followed by patients aged $0-1$ months (28/92). The high prevalence of $B$. pertussis isolation observed in babies up to age 6 months is consistent with confirmed case prevalence data in Brazil. From 2007 to $2014,34 \cdot 5 \%$ of confirmed pertussis cases occurred in infants aged 0-2 months, and $22 \cdot 4 \%$ were observed in children aged 3-6 months [4]. Currently, in Brazil pertussis immunization is done with three doses of wP vaccine given at ages 2, 4 and 6 months, followed by two booster doses at ages 15 and 48 months. Thus, as in Brazil, the high prevalence of pertussis in Distrito Federal has been observed in children in the process of immunization [4].

Seroprevalence studies of anti-pertussis toxin in people demonstrated that pertussis is highly circulating in different populations with distinct vaccine profiles, including circulation in maternal age groups [5]. Thus, mother-baby contact is the most likely cause for pertussis infection during the process of immnunization or in unimmunized babies throughout the world [5]. For this reason, Brazil introduced the aP vaccination for pregnant women in 2013 [4]. In this study, the isolation of $42 \mathrm{~B}$. pertussis strains from infants aged $0-4$ months during January-August 2014 suggests the occurrence of baby infection by mother contact. However, we could not draw conclusions about the efficacy of vaccinating pregnant women because we did not have information about the mother's vaccine status. Future surveillance studies are required to demonstrate the impact of vaccination of pregnant women as a method of containing the resurgence of pertussis in Brazil.

The pattern of high prevalence of infant patients (0-4 months) observed in this study and in Brazil [4] is different from that observed in countries implementing aP vaccination. In the United States, for example, epidemic peaks with a disproportionate number of cases in teenagers (11-19 years) [10] have been observed and were attributed to the waning immunity of aP vaccine. In Brazil, the high prevalence of pertussis in infants has been attributed to the contact of unimmunized babies with subclinical adult patients [4].

All the three $B$. pertussis serotypes were identified in the isolates. Serotype 1,2 was the most frequent (49/92), followed by serotype 1,3 (30/92) and serotype $1,2,3(12 / 92)$. One strain did not present agglutination with Fim 2 and Fim 3 antibodies and was classified as not typed (Supplementary Fig. S1). During the period analysed, an inversion of serotype prevalence was observed: from August to December 2012, the most frequent serotype was 1,3 (13/17); in 2013 the frequency of serotype 1,3 decreased (13/30), and from January 2014 to August 2014 the most frequent serotype was 1,2 (33/45) (Fig. 1). Shift in serotype prevalence have already been reported in Finland between 1990 and 2003 (from 1,2 to 1,3) [11], in Poland between 1998 and 2006 (increase in 1,2) [12] and in Ireland between 2007 and 2012 (increase in 1,2 and decrease in 1,3 prevalence) [12]. Unlike Poland and Ireland, high prevalence of serotype 1,3 has been observed in countries with aP vaccine, e.g. Canada and European countries [12, 13]. As the strain used for $\mathrm{wP}$ vaccine in Brazil presents serotype 1,2,3 [14], our results suggest that the pertussis outbreaks analysed were not associated with vaccine immunity promoted by fimbrial adhesins. Adhesin characterization should be performed to evaluate this hypothesis.

The study of the evolution of $B$. pertussis is of great importance in order to understand the forces that drive the bacterium to survive and face environmental evolutions such as the pressure exerted by vaccines [15]. In this way, PFGE analysis identified 14 circulating profiles sharing $84.6 \%$ genetic similarity (Supplementary Fig. S1 and Supplementary Table S1): 11 of these profiles were observed from August to December 2012; seven in 2013 and five during January-August 2014 (Fig. 2). Prevalence of BP-XbaI0039 increased between 2012 


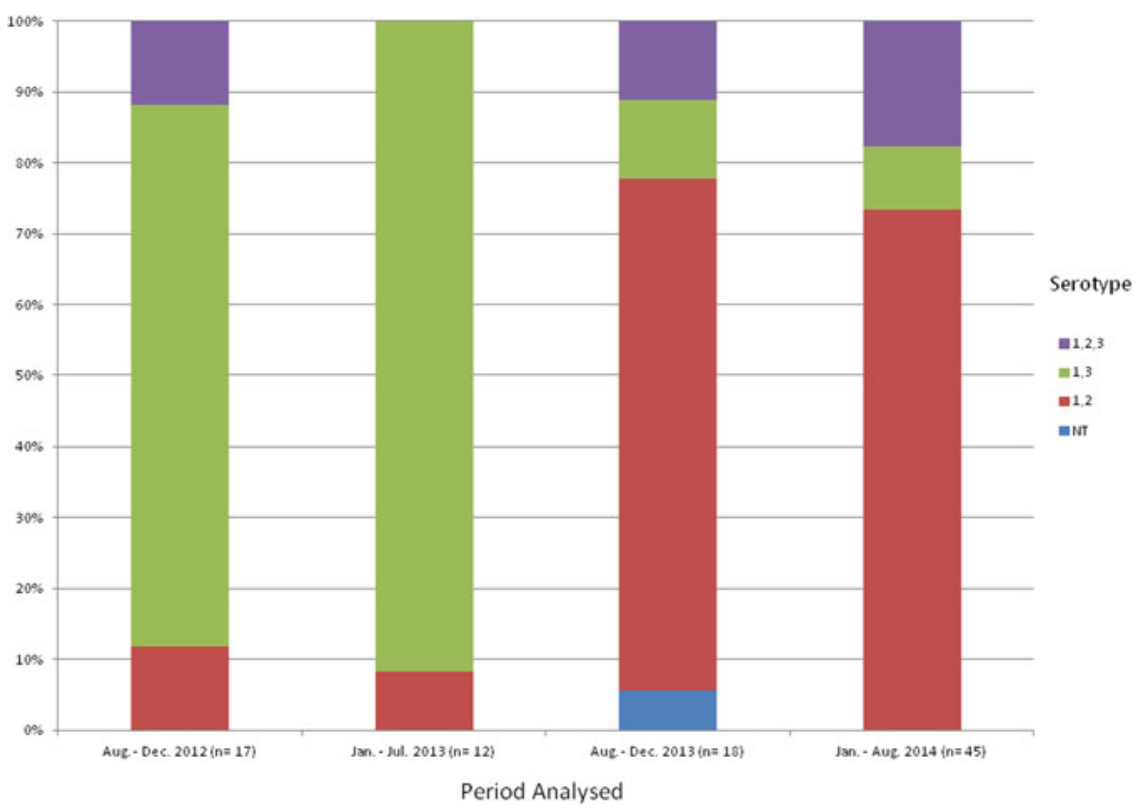

Fig. 1. Serotype prevalence of Bordetella pertussis strains isolated in the Distrito Federal region of Brazil in four periods (August-December 2012; January-July 2013; August-December 2013; January-August 2014). NT, Non-typed.

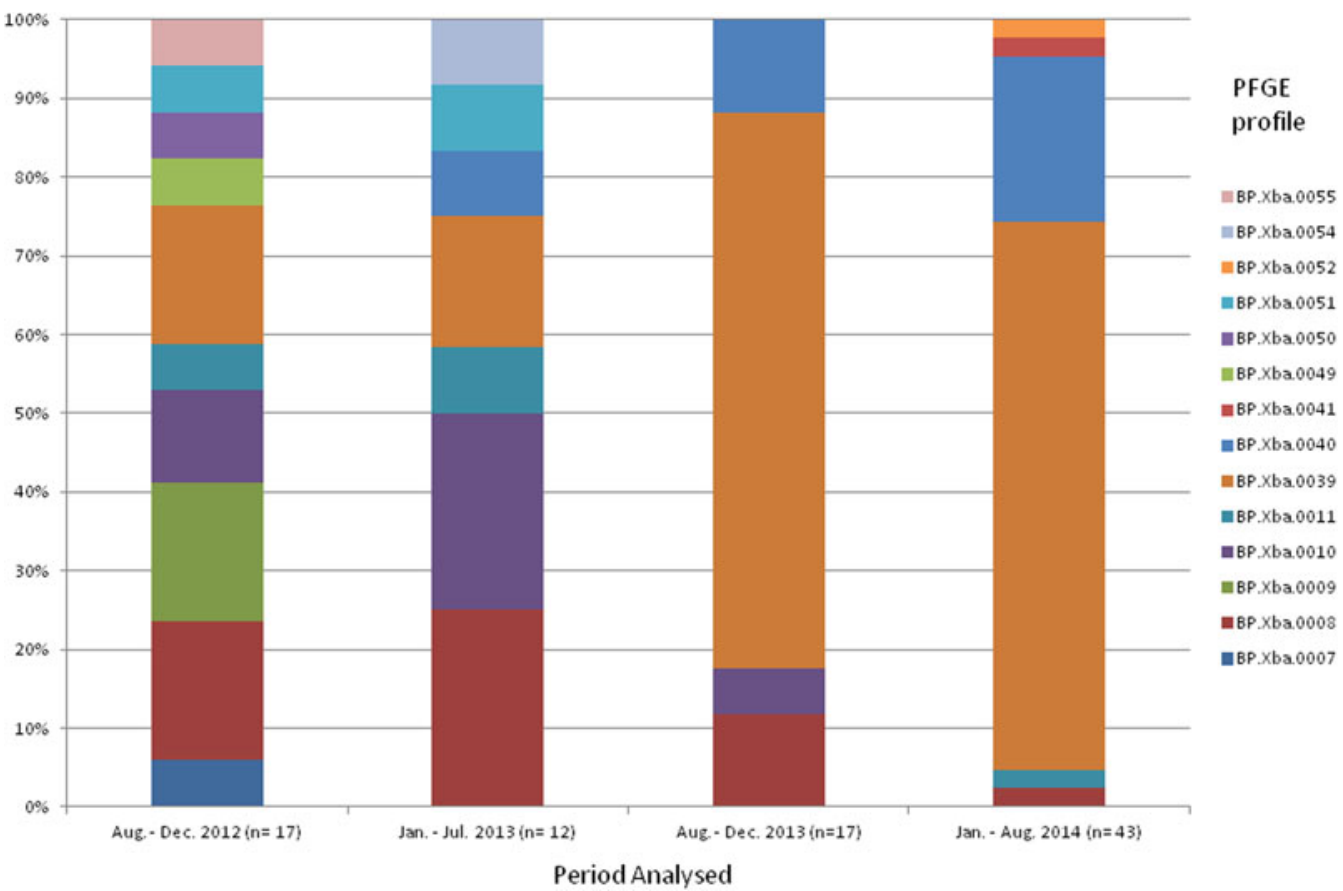

Fig. 2. Prevalence of genetic profiles identified by pulsed field gel electrophoresis (PFGE) in Bordetella pertussis strains isolated in the Distrito Federal region of Brazil in four periods (August-December 2012; January-July 2013; AugustDecember 2013; January-August 2014).

and 2014: from 17.6\% (3/17) between August and December $2012 ; 18 \cdot 2 \%(2 / 12)$ from January to July 2013 to $61 \%$ (11/18) from August to December, and 70\% (30/43) from January to August 2014 (Fig. 2). Such changes in PFGE predominant profiles as observed in our study for the BP-XbaI0039 profile or in the United States during 2000-2012 outbreaks [16] or in Europe between 1998 and 2012 [12] are probably the result of selection pressure occurring in each area considered and pertussis immunization programmes may 
contribute to this selection. As a consequence, circulating isolates differ from vaccine strains as in other countries [12, 16, 17]. The comparison of changes in predominance of PFGE profiles is relevant to monitoring the biodiversity of circulating $B$. pertussis strains. These studies are critical for understanding the epidemiology of the disease and could be a tool for optimizing prevention and control strategies for pertussis [16].

The increasing prevalence of the BP- $X b a \mathrm{I} 0039$ profile from 2012 to 2014 may suggest that BP-XbaI0039 strains are under a selection process in the region analysed. It is likely that the pertussis control programme implemented is, in some way, contributing to this. The reporting of clinical isolates with genetic and phenotypic profiles different from those of vaccine strains is evidence of selection of circulating strains by the wP vaccine. In Argentina, for example, genomic and proteomic analysis demonstrated that a clinical isolate presented more similarity with a Brazilian vaccine strain than those used in Argentinian pertussis vaccination [17].

In outbreaks occurring in Finland from 1991 to 2003, the most common PFGE profiles were clearly correlated with serotypes [11]. Unlike in Finland, the statistical analysis of serotyping and PFGE observed in this study showed that BP-XbaI0039 profile selection was independent of serotype. These observations suggest that the increasing BP-XbaI0039 prevalence was not associated with fimbrial adhesins, and was probably mediated by other cellular structures or mechanisms of the bacterium for immune evasion. Previous studies in countries with wP vaccination showed that alterations in virulence factors, such as pertactin and ptx, were associated with $B$. pertussis evolution and is suggested to be vaccine driven $[11,18,19]$.

PFGE profile changes are concurrent with other recent molecular changes in B. pertussis and may be contributing to the re-emergence of pertussis. In this study, we observed the increased prevalence of one specific PFGE profile and the decrease of other profiles during 2012-2014 outbreaks in the Central-Western Region of Brazil. The results suggest a process of selection of a B. pertussis genetic profile, probably in a vaccine-driven way. Continued PFGE monitoring is critical for understanding the changing epidemiology of pertussis [16]. Further characterization is needed to identify the virulence factors and physiological features of bacteria responsible for the selection of the BP-Xba0039 genomic profile.

Since its standardization [20], PFGE has been used as the gold standard for $B$. pertussis typing and molecular epidemiology studies. However, PFGE does not allow the comparison of patterns observed by different laboratories, which makes the identification of pandemic strains difficult. Some limitations of PFGE, e.g. non-discrimination between all unrelated isolates due to non-optimal separation of DNA fragments in every part of the gel at the same time, and, due to the presence of fragments from different parts of the chromosome presenting the same size are potential biases for $B$. pertussis typing.

Nowadays, more emphasis is placed on molecular typing methods such as PFGE in tandem with other typing methods like MLVA/MLST (multilocus variable-number tandem-repeat analysis/multilocus sequence typing). However, PFGE can be useful for strain selection when genetic diversity is a desired parameter for analysis. A complete description of the molecular evolution of $B$. pertussis provided through whole-genome methods such as PFGE and sequencing may help development of the next generation of pertussis vaccines [16]. Genomic characterization of BP-Xba0039 PFGE strains can be used for understanding the evolution of circulating strains in the region analysed.

\section{SUPPLEMENTARY MATERIAL}

For supplementary material accompanying this paper visit https://doi.org/10.1017/S0950268816003332.

\section{ACKNOWLEDGEMENTS}

We thank the FAP-DF Foundation for financial support (grant no. 193·000·561/2013)

\section{DECLARATION OF INTEREST}

None.

\section{REFERENCES}

1. Hegerle N, Guiso N. Epidemiology of whooping cough and typing of Bordetella pertussis. Future Microbiology 2013; 8: 1391-1403.

2. Guiso N. Bordetella pertussis: Why is it still circulating? Journal of Infection 2014; 68: 119-24.

3. Nitsch-Osucha A, et al. Epidemiological and immunological reasons for pertussis vaccination in adolescents and adults. Respiratory Physiology and Neurobiology 2013; 187: 99-103.

4. Guimarães LM, Carneiro ELNC. Increasing incidence of pertussis in Brazil: a retrospective study using surveillance data. BMC Infectious Diseases 2015; 15: 442. 
5. Barkoff MA, Gröndahl-Yli-Hannuksela K, He Q. Seroprevalence studies of pertussis: what have we learned from different immunized populations. FEMS Pathogens and Diseases. Published online: 24 July 2015. doi: 10.1093/femspd/ftv050.

6. Cherry JD. Historical perspective on Pertussis and use of vaccines to prevent it: 100 years of pertussis (the cough of 100 days). Microbe 2009; 2: 139-44.

7. Leite D, et al. Serotypes and genetic profiles of Bordetella pertussis strains isolated in the city of São Paulo, 2006-2008. Jornal de Pediatria 2012; 88: 357-360.

8. Tsang SW, et al. Use of monoclonal antibodies to serotype Bordetella pertussis isolates: comparison of results obtained by indirect whole-cell enzyme-linked immunosorbent assay and bacterial microagglutination methods. Journal of Clinical Microbiology 2005; 43: 2449-2451.

9. Advani A, Donnelly D, Hallander H. Reference system for characterisation of Bordetella pertussis pulsed-field gel electrophoresis profiles. Journal of Clinical Microbiology 2004; 42: 2890-2897.

10. Lavine $\mathbf{J}$, et al. Imperfect vaccine-induced immunity and whooping cough transmission to infants. Vaccine 2010; 29: 11-16.

11. Ellomaa A, et al. Strains variation among Bordetella pertussis isolates in Finland, where the whole-cell pertussis vaccine has been used for 50 years. Journal of Clinical Microbiology 2005; 43: 3681-3687.

12. Gent M, et al. Analysis of Bordetella pertussis clinical isolates circulating in Eruopean countries during the period 1998-2012. European Journal of Clinical Microbiology and Infectious Diseases 2015; 34: 821-830.
13. Simmonds K, et al. Dominance of two genotypes of Bordetella pertussis during a period of increased pertussis activity in Alberta, Canada: January to August 2012. International Journal of Infectious Diseases 2014; 29: $223-225$

14. Akamatsu MA, et al. Whole-genome sequence of a Bordetella pertussis Brazilian vaccine strain. Genome Announcements 2015; 3: e01570-14.

15. Bouchez V, Guiso N. Bordetella pertussis, Bordetella parapertussis, vaccines and cycles of whooping cough. FEMS Pathogens and Diseases. Published online: 4 August 2015. doi: 10.1093/femspd/ftv057.

16. Cassiday PK, et al. Changes in predominance of pulsed-field gel electrophoresis profiles of Bordetella pertussis isolates, United States, 2000-2012. Emerging Infectious Diseases 2016; 22: 442-448.

17. Bottero D, et al. Genotypic and phenotypic characterization of Bordetella pertussis strains used in different vaccine formulations in Latin America. Journal of Applied Microbiology 2012; 112: 1266-1276.

18. Van Loo IH, Mooi FR. Changes in the Dutch Bordetella pertussis population in the first 20 years after the introduction of whole-cell vaccine. Microbiology 2002; 148: 2011-2018.

19. Weber C, et al. Polymorphism of Bordetella pertussis isolates circulating for the last 10 years in France, where a single effective whole-cell vaccine has been used for more 30 years. Journal of Clinical Microbiology 2001; 39: 4396-4403.

20. Hardwick TH, et al. Reproducibility of Bordetella pertussis genomic DNA fragments generated by XbaI restriction and resolved by pulsed-field gel electrophoresis. Journal of Clinical Microbiology 2002; 40: 811-816. 